outcomes can be achieved with most patients spared surgery. Minor recurrence occurs frequently but can be successfully managed with close surveillance.

Disclosure of Interest None Declared.

\section{PTH-071 THE IMPACT OF INTRODUCING THE 24/7 EMEGRENCY GASTROINTESTINAL BLEED SERVICE ON REDUCING HOSPITAL STAY AND MORTALITY IN A DISTRICT GENERAL HOSPITAL}

doi:10.1136/gutjnl-2013-304907.558

1."Z U Rahman, 'B Hitchcock, 'N Mcgrath, ${ }^{2} \mathrm{~S}$ Ahmed, 'A Dixon. ' Gastroenterology; ${ }^{2}$ Kettering General Hospital, Kettering, UK

Introduction Upper Gastrointestinal (GI) bleed remains the commonest gastrointestinal emergency with a significantly high hospital mortality and prolonged hospital stay[1]. The aim of the study is to audit any difference of the above parameters after the introduction of GI bleed rota in our trust.

Methods The data was collected from patient records and endoscopy database. Rockall scores were used for risk stratification. Standard statistical methods were used for analysing data. Comparisons were made with the audit results from 2005.

Results The re audit was conducted as a follow up to the one which was done in 2005, after introducing local upper GI bleed guidelines and 24/7 GI bleed rota.

A total of 107 patients (including both in- and outpatients) were referred for upper Gastrointestinal endoscopy with suspected upper GI bleed from $1^{\text {st }}$ Jan to $30^{\text {th }}$ June 2012 . A sample of 39 patients were randomly (every third patient) included in the audit with 19 females and 20 males. The mean age was 69.6yrs ( $\pm 30 \mathrm{yrs}$ ).

The time interval from presentation to therapeutic endoscopy was 35.35 hours as opposed to 2.82 days according to 2005 audit data. Amongst the endoscopy findings $12.82 \%$ (5/39) patients had Gastrooesophageal varices versus no banding in 2005 audit data; there was no significant difference amongst other aetiologies in both audit samples. Thirty day mortality was 7.7\%(3/39) as compared to $13.33 \%(4 / 30)$ in 2005 . The length of hospital stay was found to be 10.7 days as compared to 12.32 days (2005).

Conclusion

1. The mortality was reduced as the time delay to therapeutic endoscopy reduced.

2. The hospital stay has been shortened by a couple of days in this study sample. The estimated cost of $24 / 7$ GI bleed rota is 15000 pounds per annum which can potentially save significant amount of funding by reducing hospital stay i.e. An investment worth spending on.

3. The incidence of variceal bleeding has increased significantly over the years.

Disclosure of Interest None Declared.

\section{REFERENCE}

1. Rockall TA, Logan RFA, Devlin HB, et al. Incidence of and mortality from acute upper gastrointestinal haemorrhage in the United Kingdom. BMJ1995; 311:222-6.

\section{Health service research and IT}

\section{PTH-072 A RETROSPECTIVE REVIEW OF VIDEO CAPSULE ENDOSCOPY (VCE)}

doi:10.1136/gutjnl-2013-304907.559

1.2, B Mustafa, ${ }^{3} \mathrm{~L}$ Langmead, ${ }^{2} \mathrm{M}$ Samaan. ${ }^{1}$ Queen Mary University of London; ${ }^{2}$ Royal London hospital, London, UK; ${ }^{3}$ Gastroenterology, Royal London hospital, London, UK

Introduction Background: Video capsule endoscopy (VCE) is one of the main investigations of the small bowel, especially in areas that cannot be examined by conventional endoscopy. VCE is considered gold standard for investigating obscure gastrointestinal bleeding and iron deficiency anaemia (IDA).

Methods Patients who had VCE at our hospital over two separate periods of time were included. Case notes and VCE results were reviewed. In between the two time periods patency capsule became available at the hospital and new BSG guidelines for management of IDA were published. We compared the two time periods for indication with particular reference to adherence to the IDA guidelines, and capsule retention.

Results 233 patients were identified, 48\% ( $\mathrm{n}=111)$ male and $52 \%$ $(\mathrm{n}=122)$ female. Median age (range) was 51 years (7-94) with majority $30-80$ years.

Indications IDA was the commonest indication for VCE (52.3\%), followed by exclusion of inflammatory bowel disease (IBD) (21\%). No difference was seen in referral patterns for IDA despite the IDA guidelines.

Findings The most common finding overall was angioectasia (39.4\%), of which $71 \%$ had IDA as indication. IBD was diagnosed in $4.2 \%$. In cases of IDA, angioectasia was the most common finding (56\%). There were no significant differences in findings between the two years studied. Non-steroidal anti-inflammatory drugs (NSAIDs) were more commonly used by patients referred with IDA $(p=0.009)$ but did not have an effect on the VCE result.

Patency capsule was available only for the second period. It was performed in 22 (18.6\% of referrals) cases. More than half of them were referred to investigate IBD; 11 VCEs were excluded on the basis of patency capsule result. The other 11 went on to have VCE. Capsule retention was documented in three cases overall (1.3\%), one of them had prior patency capsule. Two of them passed the capsule without surgical intervention, and the third case was lost to follow up.

Conclusion VCE is most commonly used to investigate obscure gastrointestinal bleeding and IDA. It is increasingly utilised for other indications such as diagnosis and assessment of IBD and coeliac disease. BSG guidelines for management of IDA recommend VCE only in certain groups of patients. We could significantly reduce VCE workload and resource requirements by adhering more closely to these guidelines.

Patency capsule has opened the door to patients with potential strictures and probably increases opportunity to visualise the small bowel in Crohns disease particularly. However passage of the patency capsule does not $100 \%$ rule out VCE retention.

Disclosure of Interest None Declared.

\section{PTH-073 SOCIAL MEDIA USE BY INFLAMMATORY BOWEL DISEASE AND VIRAL HEPATITIS PATIENTS AND POTENTIAL APPLICATION FOR HEALTHCARE}

doi:10.1136/gutjnl-2013-304907.560

${ }^{1} \mathrm{C}$ Timms, 1,"D Chan, ${ }^{1} \mathrm{~J}-\mathrm{Y}$ Kang, ' $\mathrm{D}$ Forton, ${ }^{1} \mathrm{~A}$ Poullis. 'Department of Gastroenterology, St George's Hospital, London, UK

Introduction Currently, inflammatory bowel disease (IBD) and viral hepatitis management involve regular visits to hospital clinics. This requires frequent time taken out of work and family life to attend. This study investigates patients' current social media use and attitude toward the potential role social media could have in reducing their hospital visits.

Methods Over a one month period (November 2012), IBD and hepatitis B and C patients attending the Gastroenterology and Hepatology Outpatient Departments of St. George's Hospital were identified. Convenience sampling was used; all IBD and hepatitis B and $\mathrm{C}$ patients encountered in these clinics were asked to participate. Patients were asked to complete a questionnaire, gathering information about their disease, current use of social media and their views about social media in the management of their disease 
Results 180 patients participated in the study: 95 patients were male and 85 were female. The combined mean age was 38.9 years (range of 15-85 years). 112 patients had IBD and 68 had viral hepatitis. The mean age for the IBD patients was 39 years (range 16-85 years) and the mean age for the hepatitis patients was 38.6 years (range 15-63 years). 46 (43.75\%) of the IBD patients and 49 (72\%) of the hepatitis patients were male.

In our study, $82.1 \%$ of the IBD and $72.1 \%$ of the viral hepatitis patients used one or more social networking site. Facebook was the most popular site. $29.5 \%$ of the IBD and $33.8 \%$ of the viral hepatitis patients used social media for support with their illness. $80.4 \%$ of the IBD patients and $72.9 \%$ of the viral hepatitis patients said they would be happy with some form of social media interaction by healthcare professionals. $84.8 \%$ of the IBD patients and $72.1 \%$ of the viral hepatitis patients were in favour of a specific social media website for their disease.

Conclusion A large proportion of patients with IBD and viral hepatitis already use social networking sites. This study suggests that the majority of both IBD and viral hepatitis patients would welcome the use of social media as part of their illness management. There are already some social media sites that have been setup for these patient groups. Increasing the awareness of these sites and further research investigating the integration of social media into the current management of both these patient groups is needed.

Disclosure of Interest None Declared.

\section{PTH-074 OF WHAT VALUE IS A 1:1 MULTIDISCIPLINARY WEIGHT MANAGEMENT CLINIC?}

doi:10.1136/gutjnl-2013-304907.561

1."D Turner, ${ }^{2} \mathrm{~S}$ Jones, ${ }^{2} \mathrm{~S}$ Syal, ${ }^{2} \mathrm{~J}$ Gray, ${ }^{2} \mathrm{~J}$ Reynolds, ${ }^{2} \mathrm{~A}$ Weaver, ${ }^{2} \mathrm{~N}$ Haboubi. ${ }^{1} \mathrm{Cardiff}$ Medical School, Cardiff; ${ }^{2}$ Aneurin Bevan Weight Management Clinic, Ebbw Vale, UK

Introduction Obesity management in Wales includes provision of a 1:1 Multidisciplinary Weight Management Clinic (MDWMC). Strategic management of obesity in Wales is guided by The All Wales Obesity Pathway and recommends MDWMCs for people with obesity who have one or more co-morbidities and who have tried several interventions without success, or who have complex emotional relationships with food. ${ }^{1}$ This service evaluation aimed to assess physiological benefits associated with attendance at a MDWMC. It also aimed to collect qualitative data in an attempt to explain any reasons for achieving benefits.

Methods An approved questionnaire was used to conduct semistructured interviews with 180 patients attending the MDWMC at Aneurin Bevan Hospital, Ebbw Vale, Wales. Quantitative data were tabulated and a thematic analysis was performed on free-text responses to collate qualitative data.

Results The MDWMC supports weight loss with $95 \%$ of patients reporting loss. For those whom baseline data was available 73\% lost at least $5 \%$ of initial body weight. $88 \%$ of patients prefer individual appointments and over $90 \%$ of patients who see each team member find consultations useful. $69 \%$ of patients report improved health mainly due to a decrease in obesity-related symptoms, and of patients taking obesity-related medication $48 \%$ report a reduction in dose of medication for asthma, $42 \%$ report a reduction in dose of antidepressants and $36 \%$ report a reduction in dose for medication for diabetes. Of employed patients $30 \%$ report a reduction of days taken off work due to sickness. $96 \%$ of patients would recommend the clinic to others. Conclusion A 1:1 Multi-disciplinary Weight Management Clinic provides value in reducing obesity and symptoms of obesity-related diseases. It also is a treatment choice favoured by patients.

Disclosure of Interest None Declared.

\section{REFERENCE}

Welsh Assembly Government. All Wales Obesity Pathway. 2009. Accessed at: http://wales.gov.uk/topics/health/improvement/index/pathway/?lang = en

\section{PTH-075 YOU'VE GUT MAIL: A GASTROENTEROLOGY EMAIL HELPLINE IS EFFICIENT AND COST-EFFECTIVE IN RESOLVING PATIENT QUERIES AND REDUCING NON- ELECTIVE INPATIENT BED DAYS}

doi:10.1136/gutjnl-2013-304907.562

1."J L S Ooi, 'M Vinayaga-Pavan, 'D C K Koh, 'V Morgan, 'V S Wong. 'Gastroenterology, Whittington Health NHS Trust, London, UK

Introduction Electronic patient-provider communication may be a convenient, cost-effective complement to standard healthcare services $^{1}$. We piloted a dedicated gastroenterology (GI) email helpline to increase accessibility for patients and medical colleagues seeking specialist advice.

Methods Retrospective study of activity records of our GI specialist nurse-led email helpline across 40 months (Jan 2008-Jul 2010; Feb-Dec 2012). Data for 2012 was analysed to demonstrate efficiency. From our highest frequency user group - inflammatory bowel disease (IBD) patients - we identified a cohort of 21 users that had contact with our department 6 months before their index email, comparing hospital utilisation rates then and in the 6 months afterward (post-intervention). Analysis was by Mann-Whitney and $\chi^{2}$ tests. Cost savings were estimated based on Department of Health Reference Costs 2011/12.

Results The helpline received 264 emails from 153 users over 40 months: $73 \%$ from patients, $20 \%$ from GPs, $7 \%$ from others. Of 126 patients, $57 \%$ were female, $43 \%$ male. Mean age was 42.4 years (range 18-82 years). 2012 data analysis: Of 72 email queries, $72 \%$ were successfully resolved electronically. Mean turnaround time was 2.2 working days (range 0-9). 69\% (50 emails) concerned general advice, medications or results, queries that are conventionally handled in outpatients (OP; £141/appointment) or in telephone clinic (TC; $£ 55 /$ appointment). Only $8.5 \%$ of email queries subsequently required TC encounters; another $10 \%$ proceeded to OP. By approximating 1 hour's work per week for a GI specialist nurse at $£ 22 /$ hour $^{2}$, we estimate the email service cost $£ 1144$ in 2012 , plus $£ 1317$ for ensuing TCs and OPs, a total of £2461. This compares favourably to $£ 2750$ to answer the 50 queries by TC alone, or $£ 7050$ by $\mathrm{OP}$ alone. Highest uptake was among the IBD subgroup: 49 users generated 129 emails over 40 months. In the 6 months pre- \& postindex email, our identified cohort $(n=21)$ had similar rates of clinic attendance (41 vs 53 appts, $p>0.05$ ), DNAs ( 3 vs 3 ) and A\&E attendance ( 4 vs 0 visits, $p>0.05$ ). Reduction in non-elective inpatient bed days was significant (34 vs 4 days, $p<0.0001$; $£ 271 /$ day), representing savings of $£ 8130$ over 6 months.

\section{Reasons behind Email Queries}

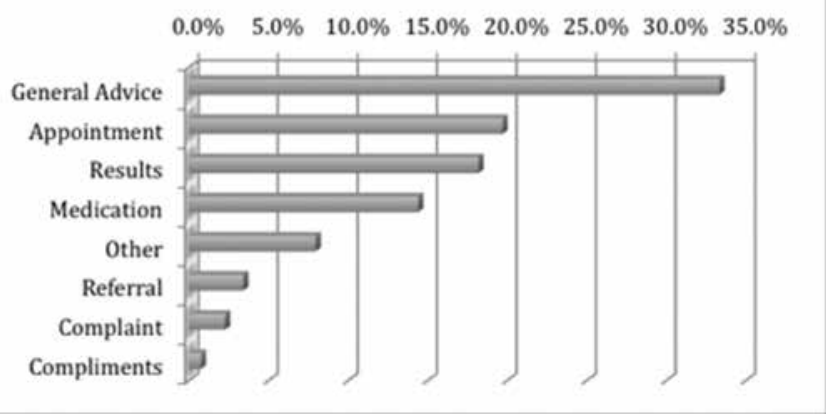

\section{Abstract PTH-075 Figure 1}

Conclusion Our GI email helpline has proven to be popular and economical. Most queries were resolved electronically, significantly reducing unscheduled inpatient bed days. We are planning a user 